\title{
ON THE DECOMPOSABILITY OF LINEAR COMBINATIONS OF EULER POLYNOMIALS
}

\author{
ÁKOS PINTÉR AND CSABA RAKACZKI
}

Received 01 July, 2016

\begin{abstract}
Considering the effect of addition to the polynomial decomposition, we present some results on the decomposability of linear combinations of Euler polynomials.
\end{abstract}

2010 Mathematics Subject Classification: 11B68

Keywords: Euler polynomials, decomposition of polynomials

\section{INTRODUCTION}

Let $\mathbb{K}$ be a field. If $f(x) \in \mathbb{K}[x]$ has degree at least 2 , we say that $f(x)$ is decomposable over the field $\mathbb{K}$ if we can write $f(x)=f_{1}\left(f_{2}(x)\right)$ for some nonlinear polynomial $f_{1}(x), f_{2}(x) \in \mathbb{K}[x]$. Otherwise, we say that $f(x)$ is indecomposable over $\mathbb{K}$. Two decompositions $f(x)=f_{1}\left(f_{2}(x)\right)$ and $f(x)=F_{1}\left(F_{2}(x)\right)$ are said to be equivalent over the field $\mathbb{K}$, written $f_{1} \circ f_{2} \sim_{\mathbb{K}} F_{1} \circ F_{2}$, if there exists a linear polynomial $l(x) \in \mathbb{K}[x]$ such that

$$
f_{1}(x)=F_{1}(l(x)) \text { and } F_{2}(x)=l\left(f_{2}(x)\right) .
$$

For a given $f(x) \in \mathbb{K}[x]$ with degree at least 2, a complete decomposition of $f(x)$ over $\mathbb{K}$ is a decomposition $f=f_{1} \circ \cdots \circ f_{k}$, where the polynomials $f_{i} \in \mathbb{K}[x]$ are indecomposable over $\mathbb{K}$ for $i=1, \ldots, k$. A polynomial of degree greater than 1 always has a complete decomposition. However, it is not unique even up to equivalence.

Euler polynomials are defined by the following generating function

$$
\sum_{n=0}^{\infty} E_{n}(x) \frac{t^{n}}{n !}=\frac{2}{e^{t}+1} e^{t x} .
$$

Euler polynomials appear in statistical physics as well as in semi-classical approximations to quantum probability distributions (see [2]), in various approximation and

Supported in part by the Hungarian Academy of Sciences, and OTKA grants K100339, NK101680, NK104208. This research was partially carried out in the framework of the Center of Excellence of Mechatronics and Logistics at the University of Miskolc. 
expansion formulas in discrete mathematics and in number theory (see for instance [1], [4]), in $p$-adic analysis (see [8], Chapter 2).

In their paper, Kreso and Rakaczki [9] proved that every Euler polynomial with odd degree is indecomposable, while if the degree is even, they characterized all the possible decompositions. For further results for the decomposability of an infinite family of polynomials we refer to [3], [6] and [5].

The purpose of this note is to investigate the decomposability of the family of polynomials $P_{n}(x)$ generated by the following function

$$
\sum_{n=0}^{\infty} P_{n}(x) \frac{t^{n}}{n !}=g(t) e^{t x},
$$

where

$$
g(t)=\frac{p(t)}{e^{t}+1}=\frac{a_{k} t^{k}+\cdots+a_{1} t+a_{0}}{e^{t}+1}, \quad a_{k}, \ldots, a_{0} \in \mathbb{Q} .
$$

If $p(t)=2$ we get back the Euler polynomials. From the above generating function (1.1) one can deduce that

$$
\begin{gathered}
P_{n}(x) \\
=\left\{\begin{array}{l}
\frac{a_{0}}{2}, \text { if } n=0 ; \\
\frac{a_{0}}{2} E_{n}(x)+\sum_{j=1}^{n} \frac{a_{j}}{2} n(n-1) \cdots(n-(j-1)) E_{n-j}(x), \text { if } 1 \leq n<k ; \\
\frac{a_{0}}{2} E_{n}(x)+\sum_{j=1}^{k} \frac{a_{j}}{2} n(n-1) \cdots(n-(j-1)) E_{n-j}(x), \text { if } n \geq k .
\end{array}\right.
\end{gathered}
$$

Actually, the polynomials $P_{n}(x)$ are linear combinations of Euler polynomials with rational coefficients.

One can begin with the simple case when the polynomial $p(t)=a_{2} t^{2}+a_{0}$. We obtain that

$$
P_{0}(x)=\frac{a_{0}}{2}, P_{1}(x)=\frac{a_{0}}{2} E_{1}(x) \text { and } P_{n}(x)=\frac{a_{0}}{2} E_{n}(x)+\frac{a_{2}}{2} n(n-1) E_{n-2}(x)
$$

for $n \geq 2$. In this paper we prove that the polynomial

$$
P_{n}(x)=E_{n}(x)+c E_{n-2}(x), n \in \mathbb{N}
$$

is always indecomposable provided that $n$ is odd. If $n$ is even then we give a possible decomposition of $P_{n}(x)$ and formulate a conjecture about the complete decomposition of $P_{n}(x)$. Our results and the conjecture will be presented in the next Section. In Section 3 we collect the auxiliary results and lemmas. The proofs of our theorems are in Section 4.

\section{NEW RESUlts}

Theorem 1. Let $P_{n}(x)=E_{n}(x)+c E_{n-2}(x)$, where $c$ is an arbitrary rational number, $n \geq 2$ is an integer. Then the polynomials $P_{n}(x)$ are indecomposable for 
all odd $n$. In the case when the degree $n$ is even then the polynomial $P_{n}(x)$ has the following decomposition:

$$
P_{n}(x)=F\left(x^{2}-x\right),
$$

where $F(x) \in \mathbb{Q}[x]$.

We note that this theorem is a generalization of the result of Kreso and Rakaczki [9] when $n$ is odd since we get back their result taking $c=0$ in our theorem. The proof of the second part of Theorem 1 is trivial, because for even $n$, we have $E_{n}(x)=$ $\tilde{E}_{n}\left(x^{2}-x\right)$ with $\tilde{E}_{n}(x) \in \mathbb{Z}[x]$.

Let

and

$$
S^{+}=\{f(x) \in \mathbb{C}[x] \mid \quad f(x)=f(1-x)\}
$$

$$
S^{-}=\{f(x) \in \mathbb{C}[x] \mid \quad f(x)=-f(1-x)\} .
$$

Theorem 2. Let $P(x) \in \mathbb{Q}[x]$ be a monic polynomial. If $P(x) \in S^{+}$then $P(x)$ is always decomposable and has the following decomposition

$$
P(x)=F\left(x^{2}-x\right),
$$

where $F(x) \in \mathbb{Q}[x]$.

The following conjecture is based on a long and straightforward computation.

Conjecture 1. Let $n$ be an even positive integer and $c$ be an arbitrary rational number. Then every decomposition of the polynomial $P_{n}(x)=E_{n}(x)+c E_{n-2}(x)$ is equivalent to (2.1).

In [10] we proved a similar result for the linear combinations of Bernoulli polynomials, however, in Bernoulli case there are some exceptional cases.

Another conjecture based on our computational experiments, Theorem 1 above and Theorem in [10] is the next

Conjecture 2. Let $m, n$ be odd positive integers and $c$ be an arbitrary rational number. Then the polynomials $E_{m}(x)+c E_{n}(x)$ and $B_{m}(x)+c B_{n}(x)$ are indecomposable.

\section{Auxiliary Results}

Lemma 1. Let $f(x), g(x) \in \mathbb{R}[x]$ be monic polynomials with $\operatorname{deg} f(x)$, $\operatorname{deg} g(x)$ $\geq 2$ and $a \in \mathbb{R}$. Denote by mult $(\alpha, f(x))$ the multiplicity of the root $\alpha$ of the polynomial $f(x)$. Suppose that

$$
\operatorname{mult}(a / 2, g(a-x)-g(x))<\operatorname{mult}(a / 2, f(g(a-x))-f(g(x))) .
$$

Then $f^{\prime}(g(a / 2))=0$. 
Proof. Let $f(x)=x^{k}+b_{k-1} x^{k-1}+\cdots+b_{1} x+b_{0}$. By expanding $f(g(a-x))-$ $f(g(x))$ we get

$$
f(g(a-x))-f(g(x))=(g(a-x)-g(x)) H(x),
$$

where

$$
\begin{gathered}
H(x)=g(a-x)^{k-1}+g(a-x)^{k-2} g(x)+\cdots+g(a-x) g(x)^{k-2}+g(x)^{k-1}+ \\
b_{k-1}\left(g(a-x)^{k-2}+g(a-x)^{k-3} g(x)+\cdots+g(a-x) g(x)^{k-3}+g(x)^{k-2}\right) \\
+\cdots+b_{3}\left(g(a-x)^{2}+g(a-x) g(x)+g(x)^{2}\right)+b_{2}(g(a-x)+g(x))+b_{1} .
\end{gathered}
$$

We know that $H(a / 2)=0$ by the condition of the Lemma. But from (3.2) we get that $H(a / 2)=k g(a / 2)^{k-1}+(k-1) b_{k-1} g(a / 2)^{k-2}+\cdots+3 b_{3} g(a / 2)^{2}+2 b_{2} g(a / 2)+$ $b_{1}=f^{\prime}(g(a / 2))$.

Lemma 2. Let $f(x)=b_{k} x^{k}+\cdots+b_{1} x+b_{0} \in S^{+} \cup S^{-}$. Then

$$
b_{k-1}=-\frac{k}{2} b_{k} \text { and } b_{k-3}=\frac{k(k-1)(k-2)}{24} b_{k}-\frac{k-2}{2} b_{k-2} \text {. }
$$

Further, if $f(x) \in S^{+}$, then $\operatorname{deg} f(x)$ is even, while if $f(x) \in S^{-}$then $\operatorname{deg} f(x)$ is odd and $f(1 / 2)=0$.

Proof. Compare the coefficients of $x^{k}, x^{k-1}$ and $x^{k-3}$ in the equality $f(x)=$ $\pm f(1-x)$.

Lemma 3. Let $f(x), g(x) \in S^{+}$be polynomials with $g \neq 0$. Then there exist unique polynomials $q(x), r(x) \in S^{+}$such that

$$
f(x)=q(x) g(x)+r(x) \text { and } \operatorname{deg} r(x)<\operatorname{deg} g(x) .
$$

Proof. From the long division algorithm for polynomials we know that there exist unique polynomials $q(x), r(x) \in \mathbb{C}[x]$ satisfying (3.4). Since $f(x)=f(1-x)$ and $g(x)=g(1-x),(3.4)$ gives that

$$
f(x)=q(1-x) g(x)+r(1-x) .
$$

It follows from (3.4) and (3.5) that

$$
0=g(x)(q(1-x)-q(x))+r(1-x)-r(x) .
$$

If $q(1-x) \neq q(x)$ then

$$
\operatorname{deg}(g(x)(q(1-x)-q(x))) \geq \operatorname{deg} g(x)>\operatorname{deg}(r(1-x)-r(x))
$$

which contradicts (3.6). This means that $q(x) \in S^{+}$and so $r(x) \in S^{+}$.

The following two results are general theorems from the theory of decomposability. 
Lemma 4 (Kreso and Rakaczki [9]). Let $F(x) \in \mathbb{K}[x]$ be a monic polynomial such that $\operatorname{deg} F$ is not divisible by the characteristic of the field $\mathbb{K}$. Then for every nontrivial decomposition $F=F_{1} \circ F_{2}$ over any field extension $\mathbb{L}$ of $\mathbb{K}$, there exists a decomposition $F=\tilde{F}_{1} \circ \tilde{F}_{2}$ such that the following conditions are satisfied

- $\tilde{F}_{1} \circ \tilde{F}_{2}$ and $F_{1} \circ F_{2}$ are equivalent over $\mathbb{L}$,

- $\tilde{F}_{1}(x)$ and $\tilde{F}_{2}(x)$ are monic polynomials with coefficients in $\mathbb{K}$,

- coeff $\left(x^{\operatorname{deg} \tilde{F}_{1}-1}, \tilde{F}_{1}(x)\right)=0$.

Moreover, such decomposition $\tilde{F}_{1} \circ \tilde{F}_{2}$ is unique.

Lemma 5 (I. Gusić [7]). Let $\mathbb{K}$ be a field of characteristic zero. Suppose that the nonconstant polynomials $g, h, G, H \in \mathbb{K}[x]$ satisfy $g \circ h=G \circ H$ and $\operatorname{deg} h=\operatorname{deg} H$. Then there exists $a, b \in \mathbb{K}$. such that

$$
H(x)=a h(x)+b, G(x)=g\left(\frac{1}{a} x-b\right) .
$$

Lemma 6. Let $P(x) \in \mathbb{Q}[x]$ be a monic polynomial. Assume that $P(x) \in S^{-}$and $P(x)=f(g(x))$, where $f(x), g(x) \in \mathbb{Q}[x]$ and $\operatorname{deg}(f(x)), \operatorname{deg}(g(x))>1$. Then we can assume that $f(x), g(x)$ are monic, $g(x) \in S^{-}$and $f(x)=-f(-x)$.

Proof. From Lemma 4 we can assume that $f(x), g(x) \in \mathbb{Q}[x]$ are monic and coeff $\left(x^{\operatorname{deg} f(x)-1}, f(x)\right)=0$. Let $f(x)=x^{k}+b_{k-2} x^{k-2}+\cdots+b_{1} x+b_{0}$ and $g(x)=x^{t}+c_{t-1} x^{t-1}+\cdots+c_{1} x+c_{0}$. Since

$$
f(g(x))=P(x)=-P(1-x)=-f(g(1-x)),
$$

from Lemma 5 we obtain that there exist $a, b \in \mathbb{Q}$ such that

$$
g(x)=a g(1-x)+b, f(x)=-f\left(\frac{1}{a} x-b\right) .
$$

It follows from $\operatorname{deg} P(x)=k t$ and Lemma 2 that $k$ and $t$ are odd. Investigation of the coefficient of $x^{t}$ in the equality $g(x)=a g(1-x)+b$ yields that $1=(-1)^{t} a=-a$. Now, comparing the coefficient of $x^{k-1}$ in the equality $f(x)=-f(-x-b)$ we obtain that $0=k b$, that is $b=0$. This proves our assertion.

Finally, we need the following lemma, in which we collect some well known properties of the Euler polynomials which will be used in the sequel, sometimes without particular reference.

\section{Lemma 7.}

(a) $E_{n}(x)=(-1)^{n} E_{n}(1-x)$;

(b) $E_{n}(x+1)+E_{n}(x)=2 x^{n}$;

(c) $E_{n}^{\prime}(x)=n E_{n-1}(x)$;

(d) $E_{2 n-1}(1 / 2)=E_{2 n}(0)=E_{2 n}(1)=0$ for $n \in \mathbb{N}$;

(e) $E_{n}(x)=\sum_{k=0}^{n}\left(\begin{array}{c}n \\ k\end{array}\right) E_{k}(0) x^{n-k}$;

(f) $E_{2 n-1}(0) E_{2 n+1}(0)<0$ for $n \in \mathbb{N}$. 
Proof. See [4].

\section{Proofs of The Theorems}

Proof of Theorem 2. We prove the assertion by using induction on $n$, the degree of the polynomial $P(x)$. If $n=0$, that is $P(x)$ is a constant polynomial then the assertion is trivial. Now assume that the result is true for polynomial of degree less than $n$. From Lemma 3 we can deduce that $P(x)=q(x)\left(x^{2}-x\right)+r(x)$, where $q(x), r(x) \in S^{+}$and $\operatorname{deg} r(x)<2$. But this means that $r(x)$ is a constant polynomial and $P(x)=q(x)\left(x^{2}-x\right)+p_{0}$, where $p_{0}$ is a rational number. By the induction hypothesis, $q(x)=Q\left(x^{2}-x\right)$ with $Q(x) \in \mathbb{Q}[x]$, and thus our statement is proved.

Proof of Theorem 1. Let $n$ be odd. From the part (a) of Lemma 7 we have $P_{n}(x) \in S^{-}$. Suppose that $P_{n}(x)=f(g(x))$. From Lemma 6 we can assume that $f(x), g(x) \in \mathbb{Q}[x]$ are monic, $g(x) \in S^{-}, f(x)=-f(-x)$. Let

$$
\begin{aligned}
& f(x)=x^{k}+b_{k-2} x^{k-2}+b_{k-4} x^{k-4}+\ldots+b_{3} x^{3}+b_{1} x, \\
& g(x)=x^{t}+c_{t-1} x^{t-1}+\ldots+c_{1} x+c_{0} .
\end{aligned}
$$

Using (b) of Lemma 7 one can deduce that

$$
f(g(x+1))+f(g(x))=P_{n}(x+1)+P_{n}(x)=2 x^{n}+2 c x^{n-2} .
$$

Since $P_{n}(x) \in S^{-}$thus $P_{n}(x+1)=-P_{n}(-x)$. From (4.1) we infer that the polynomial $g(x)-g(-x)$ divides the polynomial $P_{n}(x)-P_{n}(-x)=2 x^{n}+2 c x^{n-2}$ and so one of the cases

$$
g(x)-g(-x)= \begin{cases}(i) & d x^{s} \\ \text { (ii) } & d x^{s}(x-e), \text { where } e \in\{ \pm \sqrt{c}\} \\ \text { (ii i }) & d x^{s}\left(x^{2}+c\right)\end{cases}
$$

holds. We know that

$$
g(x)-g(-x)=2 x^{t}+2 c_{t-2} x^{t-2}+\cdots+2 c_{3} x^{3}+2 c_{1} x .
$$

Consider the above three cases in (4.2). The case (ii) is simple. In this case $x=e$ is a root of the odd polynomial $g(x)-g(-x)$, but then $x=-e$ must be also a root which only possible if $c=0$. If $c=0$ we get back the case $(i)$.

In the case $(i)$ we have $d=2, s=t$ and

$$
c_{t-2}=c_{t-4}=\cdots=c_{3}=c_{1}=0
$$

by (4.3). Since the polynomial $f(x)$ is odd, it is easy to see that the coefficient of the monomial $x^{n-4}$ depends only on the term $g(x)^{k}$ in $f(g(x))=g(x)^{k}+$ $b_{k-2} g(x)^{k-2}+\cdots+b_{1} g(x)$. By expanding $g(x)^{k}$ and using (4.4) and Lemma 2 we get that this coefficient is

$$
\frac{t^{4}}{16}\left(\begin{array}{l}
k \\
4
\end{array}\right)-\frac{t}{4}\left(\begin{array}{l}
k \\
2
\end{array}\right)\left(\begin{array}{l}
t \\
3
\end{array}\right)
$$


On the other hand the coefficient of $x^{n-4}$ of $P_{n}(x)=E_{n}(x)+c E_{n-2}(x)$ is 0 by (d) and (e) of Lemma 7 . Thus we get the following equation

$$
0=\frac{t^{4}}{16}\left(\begin{array}{l}
k \\
4
\end{array}\right)-\frac{t}{4}\left(\begin{array}{l}
k \\
2
\end{array}\right)\left(\begin{array}{l}
t \\
3
\end{array}\right)=\frac{t^{2} k(k-1)}{384}\left(t^{2}(k-2)(k-3)-8(t-1)(t-2)\right) .
$$

From this one can deduce that $t$ divides $8(t-1)(t-2)$ which is impossible because $t \geq 3$ is an odd integer.

Investigate now the case ( i i i ). It is easy to see that $d=2$ and $s=t-2$. If we assume that $t>3$ then we get from (4.3) that $c_{1}=g^{\prime}(0)=0$ and $c_{t-2}=c$. It is follows from

$$
f(g(x))-f(g(-x))=2 x^{n}+2 c x^{n-2} \text { and } n>t \geq 3
$$

that

$$
n-2=\operatorname{mult}(0, f(g(x))-f(g(-x)))>\operatorname{mult}(0, g(x)-g(-x))=t-2 .
$$

By Lemma 1 one can obtain that $f^{\prime}(g(0))=0$. From

$$
P_{n}^{\prime}(x)=n E_{n-1}(x)+c(n-2) E_{n-3}(x)=f^{\prime}(g(x)) g^{\prime}(x)
$$

we infer that $x=0$ is a zero of $P_{n}^{\prime \prime}(x)$, that is

$$
0=n(n-1) E_{n-2}(0)+c(n-2)(n-3) E_{n-4}(0) .
$$

After a simple rearrange we obtain from $(f)$ of Lemma 7 that

$$
c=-\frac{n(n-1) E_{n-2}(0)}{(n-2)(n-3) E_{n-4}(0)}>0 .
$$

On the other hand, if we compare the coefficient of $x^{n-2}$ in the equality $P_{n}(x)=$ $f(g(x))$ we have that

$$
c=k c_{t-2}+\left(\begin{array}{l}
k \\
2
\end{array}\right) c_{t-1}^{2}=c k+\left(\begin{array}{l}
k \\
2
\end{array}\right) \frac{t^{2}}{4} .
$$

But this means that

$$
c=\frac{1}{1-k}\left(\begin{array}{l}
k \\
2
\end{array}\right) \frac{t^{2}}{4}=-k \frac{t^{2}}{8}<0,
$$

which contradicts (4.8).

In the remaining case when $t=\operatorname{deg} g(x)=3$ one can deduce from $g(x) \in S^{-}$, $c_{t-2}=c$ and Lemma 2 that

$$
g(x)=x^{3}-\frac{3}{2} x^{2}+c x+\frac{1}{4}-\frac{1}{2} c .
$$


Computing the coefficients of $x^{n-4}$ in the equality $E_{n}(x)+c E_{n-2}(x)=f(g(x))$ we obtain that

$$
0=\frac{81}{16}\left(\begin{array}{l}
k \\
4
\end{array}\right)+c^{2}\left(\begin{array}{l}
k \\
2
\end{array}\right)+\frac{27}{4} c\left(\begin{array}{l}
k \\
3
\end{array}\right)-3\left(\begin{array}{l}
k \\
2
\end{array}\right)\left(\frac{1}{4}-\frac{1}{2} c\right)
$$

Combining (4.11) with (4.9) we have that

$$
0=-\frac{27}{64} k^{4}+\frac{135}{128} k^{3}+\frac{33}{128} k^{2}-\frac{57}{64} k,
$$

whence

$$
k=0,1, \frac{3}{4} \pm \frac{\sqrt{385}}{12} .
$$

In the case when $n$ is even the assertion follows from (a) of Lemma 7 and Theorem 2.

\section{REFERENCES}

[1] M. Abramowitz and I. A. Stegun, Handbook of mathematical functions with formulas, graphs and mathematical tables. United States Department of Commerce, National Bureau of Standards, 1964.

[2] L. E. Ballentine and S. M. McRae, "Moment equations for probability distributions in classical and quantum mechanics," Phys. Rev. A, vol. 58, pp. 1799-1809, Sep 1998, doi: 10.1103/PhysRevA.58.1799.

[3] Y. F. Bilu, B. Brindza, P. Kirschenhofer, Á. Pintér, R. F. Tichy, and A. Schinzel, "Diophantine Equations and Bernoulli Polynomials," Compositio Mathematica, vol. 131, pp. 173-188, 2002, doi: 10.1023/A:1014972217217.

[4] J. Brillhart, "On the Euler and Bernoulli polynomials." Journal für die reine und angewandte Mathematik, vol. 234, pp. 45-64, 1969. [Online]. Available: http://eudml.org/doc/150898

[5] A. Dujella and I. Gusić, "Decomposition of a recursive family of polynomials," Monatshefte für Mathematik, vol. 152, no. 2, pp. 97-104, 2007, doi: 10.1007/s00605-007-0459-3.

[6] A. Dujella and R. F. Tichy, "Diophantine Equations for Second-Order Recursive Sequences of Polynomials," The Quarterly Journal of Mathematics, vol. 52, no. 2, pp. 161-169, 2001, doi: 10.1093/qjmath/52.2.161.

[7] I. Gusić, "On decomposition of polynomials over rings," Galsnik Matematički, vol. 43, no. 1, pp. 7-12, 2008, doi: 10.3336/gm.43.1.02.

[8] N. Koblitz, p-adic Numbers, p-adic Analysis, and Zeta-Functions. New York: Springer-Verlag, 1984. doi: 10.1007/978-1-4612-1112-9.

[9] D. Kreso and C. Rakaczki, "Diophantine equations with Euler polynomials," Acta Arithmetica, vol. 161, no. 2, pp. 267-281, 2013, doi: 10.4064/aa161-3-5.

[10] Á. Pintér and C. Rakaczki, "On the decomposability of linear combinations of bernoulli polynomials," Monatshefte für Mathematik, vol. 180, no. 3, pp. 631-648, 2016, doi: 10.1007/s00605015-0799-3. 
Authors' addresses

Ákos Pintér

Institute of Mathematics

MTA-DE Research Group "Equations, Functions and Curves"

Hungarian Academy of Sciences and University of Debrecen

P. O. Box 12, H-4010 Debrecen, Hungary

E-mail address: apinterescience.unideb.hu

Csaba Rakaczki

Institute of Mathematics

University of Miskolc

H-3515 Miskolc Campus, Hungary

E-mail address: matrcs@uni-miskolc.hu 